\title{
Información de las usuarias y consentimiento informado en inducciones del trabajo de parto
}

Mariana Araujo Zubieta ${ }^{1}$, María Noel Firpo Gallo ${ }^{1}$ EloísaCapano Añon², María José Andrade Falconi², Diego Greif Waldman ${ }^{3}$, María Fernanda Nozar Cabrera ${ }^{4}$, Verónica Fiol Lepera ${ }^{4}$, Francisco Coppola Gonzalvez ${ }^{5}$

\section{RESUMEN:}

Objetivo: Evaluar la información que poseen las usuarias del Centro Hospitalario Pereira Rossell (CHPR) respecto a la inducción farmacológica del trabajo de parto.

Material y Métodos: Estudio transversal, prospectivo y analítico en los períodos de agosto-diciembre de 2011 y mayo-julio de 2014 en la Maternidad del Centro Hospitalario Pereira Rossell (CHPR). Se estudiaron a las usuarias sometidas a inducción del parto $(n=200)$. Se analizó mediante un cuestionario la información que poseían sobre la inducción (objetivo, proceso y complicaciones de la misma) y se realizó concomitantemente una auditoría de historias clínicas revisando la constancia en las mismas del consentimiento informado de las usuarias previo al inicio de la inducción y la indicación detallada de la inducción del parto.

Resultados: Del total de inducciones, 143 fueron con oxitocina, 32 con misoprostol y 25 con la combinación de ambos fármacos. $23 \%$ de las pacientes no conocían el objetivo de la inducción del parto; $80 \%$ desconocía el proceso de la inducción y $99 \%$ desconocía las complicaciones del mismo. En el $86 \%$ de historias, no constaba el consentimiento y en un $44 \%$ no se registró la indicación.

Conclusiones: a pesar de ser la historia clínica un documento médico-legal y la inducción del parto un procedimiento no exento de complicaciones, es destacable la falta de información brindada a la paciente. (Horiz Med 2014; 14(4): 15-18)

Palabras clave: Inducción farmacológica, trabajo de parto, consentimiento informado (Fuente: DeCS BIREME)

\section{Information to users and informed consent in labor induction}

\section{ABSTRACT}

Objective: Evaluate the information held by users at Pereira Rossell Hospital about pharmacological induction of labor. Material and Methods: Cross-sectional, prospective and analytical study from August to December 2011 and from May to July 2014, in the maternity of CHPR. 200 users undergoing induction of labor were studied. Using a questionnaire, the information they had about the induction (objective, process and complications) was assessed and concomitantly an audit of medical records reviewing the records of informed consent of the patient prior to starting the induction and the detailed indications of labor induction.

Results: Of all inductions, 143 were with oxytocin, 32 with misoprostol and 25 with the combination of both drugs. $23 \%$ of patients did not know the objective of labor induction; $80 \%$ did not know the process of induction and $99 \%$ did not know the complications it might have. In $86 \%$ of medical records they did not have the patient's consent and in $44 \%$ the indication was not recorded.

Conclusions: in spite of the medical history being a medical-legal document and the labor induction a procedure that may have complications, there is are remarkable lack of information provided to the patient. (Horiz Med 2014; 14(4): 15-18)

Key words: Pharmacological induction, labor, informed consent. (source: MeSH NLM)

\footnotetext{
Ex Residente de Clínica Ginecotocológica “A” Centro Hospitalario Pereira Rossell. Facultad de Medicina. Universidad de la República Uruguay

2 Residente de Clínica Ginecotocológica “A” Centro Hospitalario Pereira Rossell. Facultad de Medicina. Universidad de la República. Uruguay

3 Asistente de Clínica Ginecotocológica “A” Centro Hospitalario Pereira Rossell. Facultad de Medicina. Universidad de la República. Uruguay

4 Profesora Adjunta Clínica Ginecotocológica “A” Centro Hospitalario Pereira Rossell. Facultad de Medicina. Universidad de la República Uruguay

5 Profesor Agregado Clínica Ginecotocológica “A” Centro Hospitalario Pereira Rossell. Facultad de Medicina. Universidad de la República Uruguay
} 


\section{INTRODUCCIÓN}

La inducción del parto, es el inicio artificial de las contracciones uterinas para producir el borramiento y dilatación del cuello uterino. Es una intervención médica, que puede exponer a la madre y el feto a efectos adversos, por lo que está indicada cuando existe un beneficio mayor materno-fetal de interrupción de la gravidez, comparado con la prolongación de la misma.

Existe un gran número de indicaciones: tanto maternas como fetales.

Se destacan: alteraciones en la duración del embarazo, alteración del crecimiento y desarrollo fetal, rotura prematura de membranas ovulares, patología médica del embarazo y otros.

Los métodos de inducción del parto pueden ser no farmacológicos, como colocación de sondas Foley, decolamiento de membranas ovulares, amniotomía, y métodos farmacológicos, como prostaglandinas sintéticas E1 (misoprostol) y E2 (dinoprostona), y oxitocina (1).

El consentimiento informado y firmado por la paciente, debe realizarse con la suficiente antelación ante la toma de decisión de inducción del parto. En situaciones de urgencia, basta con dar la información apropiada y dejar constancia de la misma en la historia clínica para cualquier intervención.

La información sobre un procedimiento médico, es un derecho de la paciente y brindarla, una obligación del médico. Esto, está consignado tanto en Normas Jurídicas nacionales como internacionales, dándole un marco legal (2).

Como objetivos principales del estudio, se propuso: evaluar la cantidad y calidad de la información que tenían las usuarias del Centro Hospitalario Pereira Rossell, expuestas a una inducción farmacológica del trabajo de parto, sobre dicho procedimiento. Análisis en detalle del conocimiento en cuanto al objetivo de la inducción del trabajo de parto, el procedimiento en sí mismo y sus complicaciones. Concomitantemente,se analizó la constancia en la historia clínica de la indicación de la inducción del parto, y el consentimiento informado de la usuaria.

\section{MATERIAL Y MÉTODOS}

Estudio transversal, prospectivo y analítico en la Maternidad del Centro Hospitalario Pereira Rossell, en el período comprendido entre agosto-diciembre de 2011 y mayo-julio de 2014. La población, estuvo constituida por 200 usuarias sometidas a inducción del trabajo de parto farmacológico, en el período de tiempo mencionado. Las mismas que se encontraban internadas en Sala de puérperas.

Se analizó mediante un cuestionario, la información que tenían sobre la inducción (objetivo, proceso y complicaciones de la misma) y se realizó concomitantemente, una auditoría de historias clínicas revisando la constancia en las mismas del consentimiento informado de las usuarias previo al inicio de la inducción y la indicación detallada de la inducción del parto.

Las inducciones analizadas, utilizaron oxitocina intravenosa, comenzando con una dosis de $3 \mathrm{mU} /$ minuto, mientras que las inducciones con misoprostol, fueron realizadas a dosis de 25 microgramos por vía yugal o intravaginal.

\section{RESULTADOS}

En los períodos de estudio, se analizaron 200 usuarias sometidas a inducción farmacológica del trabajo de parto.Los motivos de inducción farmacológica del trabajo de parto fueron: embarazos en vías de prolongación, alteraciones del crecimiento fetal, rotura prematura de membranas ovulares y patologías médicas del embarazo.

Encuantoa losmétodosfarmacológicosde inducción, se utilizó en un $71 \%$ de los casos oxitocina. En el $16 \%$ de los casos se utilizó misoprostol, y el $13 \%$ recibió inicialmente misoprostol y luego oxitocina.

Con respecto a los resultados de la entrevista realizada en forma individualizada a las usuarias, $154(77 \%)$ conocían el objetivo de la inducción del trabajo de parto, mientras que $46(23 \%)$ no lo conocían. Tabla 1. 
Tabla 1. Inducción del trabajo de parto en CHPR. $n=200$ casos

\begin{tabular}{lrr} 
& \multicolumn{1}{c}{$\mathrm{Si}$} & \multicolumn{1}{c}{ No } \\
Conoce objetivo & $154(77 \%)$ & $46(23 \%)$ \\
Conoce proceso & $40(20 \%)$ & $160(80 \%)$ \\
Conoce complicaciones & $1(0,5 \%)$ & $199(99,5 \%)$ \\
Indicación en la historia clínica & $112(56 \%)$ & $88(44 \%)$ \\
Consentimiento en la historia clínica & $28(14 \%)$ & $172(86 \%)$
\end{tabular}

Se evaluó el conocimiento de las pacientes acerca del proceso de inducción, interrogando si conocían cuando comenzarían las contracciones uterinas dolorosas, duración del procedimiento, fármaco utilizado, vía de administración, entre otras. En este caso, únicamente el $20 \%$ de las pacientes encuestadas pudo contestar correctamente estas interrogantes.

Para finalizar la encuesta, se le preguntó a la usuaria, si conocía las posibles complicaciones de la misma. Se constató aquí, que sólo el $1 \%$ las conocía al inicio de la inducción del trabajo de parto.

Al realizar la auditoría de historias clínicas, se destaca que en un $56 \%$ de los casos, constaba la indicación de la inducción, y en un $44 \%$ no se registró. En el $14 \%$ de las historias clínicas, se registró el consentimiento de las usuarias previo a iniciar la inducción del parto.

\section{DISCUSIÓN}

El adecuado registro en la historia clínica forma parte del acto médico y, por ello, constituye un criterio de lex artis. En este sentido, la cantidad y calidad de lo registrado traduce la calidad del acto médico (3). La historia clínica es, entonces; un documento médico-legal complejo, siendo una condición necesaria para la asistencia médica y aspecto crítico de la responsabilidad médica (4).

El consentimiento informado,forma parte del acto médico y debe surgir de la relación médicopaciente, siendo un derecho del paciente y una obligación ética y legal para el médico. Tiene como contrapartida desde el médico el principio de beneficencia, que constituyen junto con la autonomía, no maleficencia y justicia los principios de la bioética (5-7).
Se trata de un acto clínico que no puede ser sustituido por otro de carácter solamente formal o administrativo $(2,4,5,8,9)$. Bergese, considera que el consentimiento informado no constituye una delegación de responsabilidades del médico al paciente, sino, por el contrario, compartir las mismas $(10,11)$. Es un fenómeno bilateral.

La información al paciente es un derecho humano fundamental protegido por instrumentos internacionales y también por la Constitución de la República Oriental del Uruguay (3). Ésta, debe ser lo suficientemente razonable para que el paciente pueda tomar una decisión válida, acorde con sus valores. Debe ser ofrecida en una forma y lenguaje claro para el paciente, de acuerdo a su nivel cultural y las posibilidades de comprender la misma (4). Se ha de informar acerca de los objetivos del acto médico, las diferentes opciones, la relación beneficio-riesgo, así como las eventuales limitaciones o secuelas posteriores (3).

En principio, el consentimiento es un "contrato consensual". Tanto la propuesta, como la aceptación de un procedimiento, pueden ser expresas o tácitas. Se puede manifestar mediante el lenguaje oral o escrito, así como; también, mediante gestos o señales (1). Aún, siendo verbal y además escrita, la información debe ser trasladada al paciente con la antelación suficiente para que pueda reflexionar sobre su decisión $(8,9)$.

La información sobre la inducción del trabajo de parto podría ser brindada en el control obstétrico de un embarazo próximo al término, o con riesgo aumentado tanto materno como fetal de requerir una inducción. Se ha generado una confusión conceptual entre consentimiento informado y consentimiento firmado. Un formulario de consentimiento firmado por el paciente no limita la responsabilidad médica. Este tipo de consentimiento firmado, carece de valor ético y jurídico en ausencia de una adecuada información y una real autonomía del paciente $(2,3,5,8,9)$. Un acto médico correctamente realizado y consentido por el paciente, excepcionalmente dará lugar a reclamos independientemente de la existencia de un formulario firmado(2-4,8,9).

La inducción del parto, así como cualquier acto médico requiere el consentimiento de las 
pacientes. Para esto, es imprescindible que conozca la indicación de este procedimiento, la medicación utilizada, el tiempo que se espera lleve este proceso, así como los posibles riesgos y complicaciones que pueda determinar a nivel materno y fetal. Con esta información y analizando los riesgos y beneficios, la paciente podrá dar su consentimiento o no sobre la realización de este procedimiento, hecho que debe constar en la historia clínica.

En conclusión, se evidenció la falta de información completa a las usuarias por parte del personal médico con respecto al procedimiento de inducción del trabajo en la población estudiada; de la misma forma, queda manifiesta la carencia de registro médico y la no existencia de consentimiento informado. En nuestro medio, no existe un consentimiento informado para la aplicación de una inducción, si bien las pautas internacionales así lo recomiendan (12).

Recomendamos continuar estudiando este aspecto, para establecer una auditoría continua de registros médicos, y formular un protocolo de consentimiento informado sobre inducción del trabajo de parto que esté disponible en los Servicios de Maternidad.

\section{Fuentes de financiamiento}

El estudio ha sido autofinanciado por los autores.

\section{REFERENCIAS BIBLIOGRÁFICAS}

1. Induction of Labour. SOGC CLINICAL PRACTICE GUIDELINE. September JOGC 2013. S7 - S11

2. Castaño P. El consentimiento informado en la responsabilidad médica. Bogotá: Temis, 1997: 461.

3. Sosa Aguerre T. Relación vinculante entre médico y paciente: el consentimiento. En Sindicato Médico del Uruguay: II Jornadas de Responsabilidad Médica (1996). Montevideo: SMU, 1998: 147-52.

4. Rodríguez Almada $\mathrm{H}$, Los aspectos críticos de la responsabilidad médica y su prevención. Rev Med Uruguay 2001; 17(1): 17-23.

5. Galán Cortés JC. Aspectos legales de la relación clínica. Madrid: Jarpyo, 2000: 166.

6. Briozzo L. La crisis como oportunidad: reformulando la relación sanitaria. Rev Med Uruguay 2007; 23: 139-141

7. Briozzo L. Apuntes para la discusión: los valores del profesionalismo y el trabajo médico. Cuadernos del CES № 2 Junio 2008; 111-118.

8. Galán Cortés JC. La responsabilidad médica y el consentimiento informado. Rev Med Uruguay 1999; 15: 5-12.

9. Rodríguez Almada H. Seudoconsentimiento informado en Uruguay. Rev Med Uruguay 2002; 18:89-93.

10. González D, Rodríguez Almada H, Berro G. Consentimiento Informado. Análisis crítico de su aplicación en un servicio quirúrgico. Rev Med Uruguay 2005; 21: 291-297.

11. Bergese C. el $99 \%$ de los juicios parte de una relación médicopaciente inadecuada. En: Sindicato Médico del Uruguay. II Jornadas de responsabilidad médica (1996). Montevideo: SMU, 1998: 125-7.

12. WHO. Recommendations for induction of labour. 2011.

\section{Conflicto de interés:}

Los autores declaran no tener ningún conflicto de interés en el trabajo realizado.

\section{Correspondencia:}

Diego Greif

Teléfono: 0059899288990

Dirección: Osorio 1321/ 703 Montevideo Uruguay

Correo electrónico: diegogreif@gmail.com
Recibido: 13 de Mayo de 2014

Aprobado: 05 de Setiembre de 2014 